The Astrophysical Journal Supplement Series, 158:118-123, 2005 May

(C) 2005. The American Astronomical Society. All rights reserved. Printed in U.S.A.

\title{
THE OPTICAL DESIGN OF THE BACKGROUND EMISSION ANISOTROPY SCANNING TELESCOPE (BEAST)
}

\author{
Newton Figueiredo, ${ }^{1}$ Marco Bersanelli, ${ }^{2}$ Jeffery Childers,${ }^{3}$ Ocleto D’Arcangelo, ${ }^{4}$ Doron Halevi, ${ }^{3}$ \\ Michael Janssen, ${ }^{5}$ Keith Kedward, ${ }^{6}$ Nicole Lemaster, ${ }^{3,7}$ Philip Lubin, ${ }^{3}$ Nazzareno Mandolesi, ${ }^{8}$ \\ Joshua Marvil, ${ }^{3,7}$ Peter Meinhold, ${ }^{3,7}$ Jorge Mejía,, 9 Aniello Mennella, ${ }^{10}$ Paolo Natoli, ${ }^{11}$ \\ Hugh O’Neil, ${ }^{3,7}$ Agenor Pina, ${ }^{1}$ Mark Pryor, ${ }^{12}$ Maura Sandri, ${ }^{8}$ Alessandro Simonetto, ${ }^{4}$ \\ Carlo Sozzi,${ }^{4}$ Camilo Tello, ${ }^{9}$ Fabrizio Villa, ${ }^{8}$ Thyrso Villela, 9 \\ Brian Williams, ${ }^{3}$ and Carlos Alexandre Wuensche ${ }^{9}$ \\ Received 2004 February 2; accepted 2004 September 28
}

\begin{abstract}
We present the optical design of the Background Emission Anisotropy Scanning Telescope (BEAST), an offaxis Gregorian telescope designed to measure the angular distribution of the cosmic microwave background radiation (CMBR) at 30 and $41.5 \mathrm{GHz}$ on angular scales ranging from $20^{\prime}$ to $10^{\circ}$. The aperture of the telescope is $1.9 \mathrm{~m}$, and our design meets the strict requirements imposed by the scientific goals of the mission: the beam size is $20^{\prime}$ at $41.5 \mathrm{GHz}$ and $26^{\prime}$ at $30 \mathrm{GHz}$, while the illumination at the edge of the mirrors is lower than $-30 \mathrm{~dB}$ for the central horn. The primary mirror is an off-axis section of a paraboloid, and the secondary an off-axis section of an ellipsoid. A spinning flat mirror located between the sky and the primary provides a two-dimensional chop by rotating the beams around an ellipse on the sky. BEAST uses a receiver array of cryogenic low noise InP High Electron Mobility Transistor (HEMT) amplifiers. The baseline array has seven horns matched to one amplifier each and one horn matched to two amplifiers (two polarizations) for a total of nine amplifiers. Two horns operate around $30 \mathrm{GHz}$, and six operate around 41.5 GHz. Subsequent campaigns will include $90 \mathrm{GHz}$ and higher frequency channels.
\end{abstract}

Subject headings: cosmic microwave background - cosmology: observations - telescopes

\section{INTRODUCTION}

Understanding the mechanisms of structure formation in the early universe $(10<z<1000)$ is one of the most important and active areas in cosmology today, and measurements of the cosmic microwave background radiation (CMBR) anisotropy play a pivotal role in this field. By measuring the angular power spectrum of CMBR fluctuations, one can discriminate among various competing theories that predict the primordial mass distribution (e.g., inflation, cosmic strings and textures, and primordial isocurvature baryonic perturbations) and understand the gravitational collapse that ultimately brought about the formation of galaxies. Since the fluctuation amplitudes at angular scales of a few degrees and smaller are also sensitive to the free electron distribution, CMBR measurements can also be used to determine the ionization history of the universe.

We have built the Background Emission Anisotropy Scanning Telescope (BEAST), a 2.2 m off-axis Gregorian telescope

\footnotetext{
1 Universidade Federal de Itajubá, Departamento de Física e Química, Caixa Postal 50, 37500-903-Itajubá, MG, Brazil; newton@unifei.edu.br.

2 Dipartimento di Fisica, Università degli study di Milano, via Celoria 16 , 20133 Milano, Italy.

3 Physics Department, University of California, Santa Barbara, CA 93106.

4 IFP-CNR, via Cozzi 53, 20125 Milano, Italy.

5 Jet Propulsion Laboratory, California Institute of Technology, Oak Grove Drive, Pasadena, CA 91109.

6 Department of Mechanical Engineering, University of California, Santa Barbara, CA 93106.

7 UC Santa Barbara Center for High Altitude Astrophysics at White Mountain, Santa Barbara, CA 93106.

8 IASF-CNR sezione di Bologna, via P. Gobetti, 101, 40129, Bologna, Italy.

9 Instituto Nacional de Pesquisas Espaciais, Divisão de Astrofísica, Caixa Postal 515, 12210-070-São José dos Campos, SP, Brazil.

${ }^{10}$ IASF-CNR, via Bassini 15, 20133 Milano, Italy.

11 Dipartimento di Fisica e sezione INFN, Università di Roma "Tor Vergata," Rome, Italy.

${ }^{12}$ Composite Optics Incorporated, San Diego, CA.
}

fed by a set of six Q-band and two Ka-band scalar feed horns, which performed two balloon flights and a ground campaign at a $3.8 \mathrm{~km}$ altitude site. A sister optics design has been already set up in the same site for polarization measurements. A full description of the instrument can be found in Childers et al. (2005), and the observational results are reported in Meinhold et al. (2005). In this paper we present a detailed description of the telescope optics.

\section{THE OPTICAL DESIGN}

In order to minimize sidelobe contamination and blockage, BEAST uses a Gregorian off-axis configuration fed by an array of eight horns on the focal plane. The primary mirror is an offaxis section of a paraboloid, and the secondary is an off-axis section of an ellipsoid. A spinning flat mirror located between the sky and the primary provides a two-dimensional chop by rotating the beams around an ellipse on the sky. Figure 1 shows a ray-tracing diagram of BEAST optics.

In order to achieve the desired goals, the optical design of the telescope must satisfy some strict requirements:

1. The beam size at $41.5 \mathrm{GHz}$ must be smaller than $21^{\prime}$ so that the experiment can accurately measure the first Doppler peak in the CMBR spectrum (e.g., Scott et al. 1995).

2. The edge illumination of the mirrors must be smaller than $-30 \mathrm{~dB}$ for both frequencies in order to avoid sidelobe contamination by the radiation due to the Earth or the balloon.

3. The geometry of the telescope must satisfy the DragoneMizuguchi condition so that the instrument can be used for polarization measurements (Dragone 1978; Mizuguchi et al. 1978).

4. The size of the primary mirror cannot exceed $2.2 \mathrm{~m}$ in order to fit inside the gondola.

It is very difficult to simultaneously satisfy these four conditions. There is a trade-off between the first two conditions, 


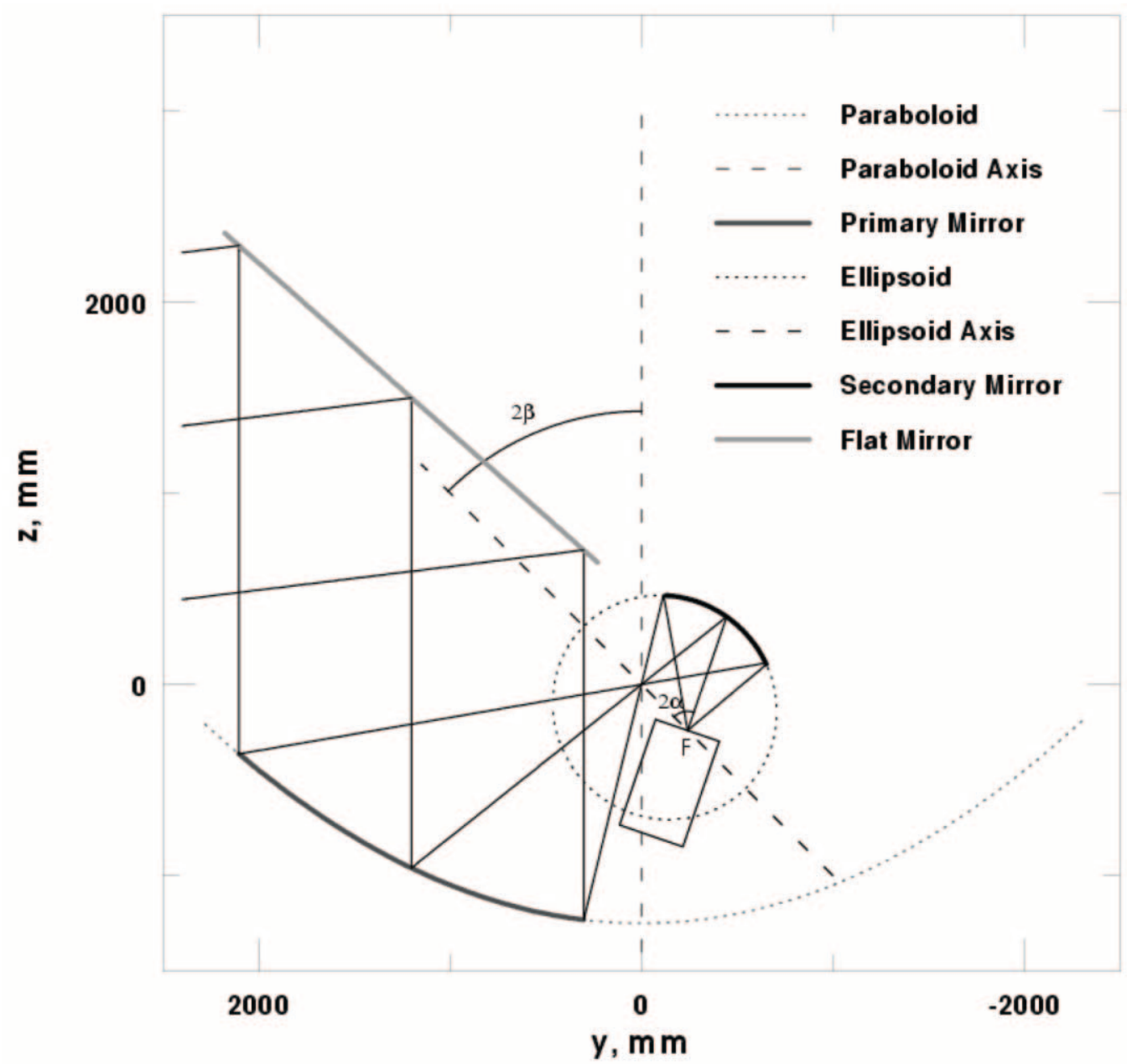

FIG. 1.-Ray-tracing diagram of BEAST optics.

since improving the illumination increases the beam size. The third and fourth conditions, on the other hand, limit significantly the allowed range of the parameters that define the optics. In the following sections we present the optimal solution we found and the final configuration of the telescope.

\subsection{Corrugated Horns}

Conical corrugated horns are employed to couple the radiation between the antenna and the detectors. This kind of feed offers two features that make it the best choice for a telescope like BEAST: it has symmetric radiation pattern and null crosspolarization (Clarricoats \& Olver 1984). Its wavefront is approximately spherical, but the position of the center of the sphere, the so-called phase center, depends on the distance to the point where the phase is measured. For a telescope with only one horn, its phase center should be at the focus of the telescope (Dragone 1978, 1981). Since BEAST has multiple horns at the focal plane, only the phase center of the central horn is at the focus. Figure 2 shows the projected aperture of the horns as they are mounted on the focal plane.

In order to calculate the position of the phase center with respect to the aperture of the central horn, we followed the procedure outlined by Ohtera \& Ujiie (1975). Table 1 lists, for each horn, the radius of its aperture $a$, its length $l$, which is given by $l=a / \tan \phi_{0}$, the distance between the phase center and the aperture (PA) and the distance between the aperture and the secondary mirror (AS). Note that $\phi_{0}=7^{\circ}$ for both horns.

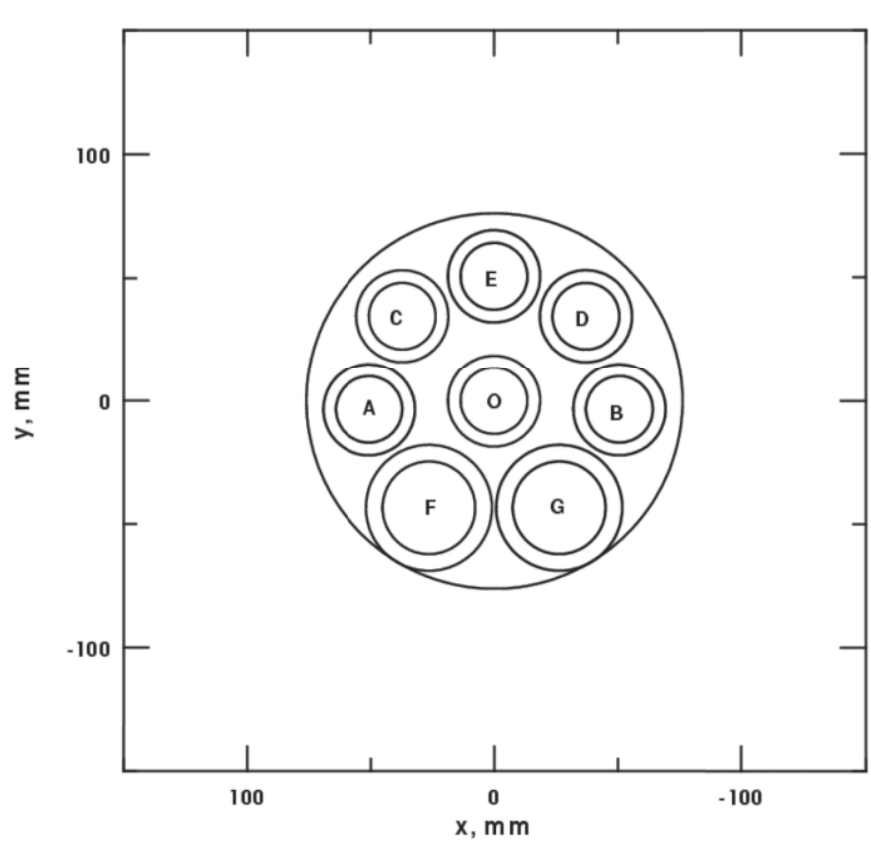

FIG. 2.-Focal plane array as seen from the secondary showing the aperture of six Q-band (A, B, C, D, E, and O) and two Ka-band (F and G) horns. 
TABLE 1

Geometric Parameters of the Horns

\begin{tabular}{crccccc}
\hline \hline $\begin{array}{c}\nu \\
(\mathrm{GHz})\end{array}$ & $\begin{array}{c}\lambda \\
(\mathrm{mm})\end{array}$ & $\begin{array}{c}a \\
(\mathrm{~mm})\end{array}$ & $\begin{array}{c}l \\
(\mathrm{~mm})\end{array}$ & $\begin{array}{c}\phi_{0} \\
(\mathrm{deg})\end{array}$ & $\begin{array}{c}\mathrm{PA} \\
(\mathrm{mm})\end{array}$ & $\begin{array}{c}\text { AS } \\
(\mathrm{mm})\end{array}$ \\
\hline $30.0 \ldots \ldots \ldots \ldots \ldots$. & 10.0 & 18.8 & 152.7 & 7.0 & 4.9 & 931.8 \\
$41.5 \ldots \ldots \ldots \ldots \ldots \ldots . .$. & 7.2 & 13.5 & 110.0 & 7.0 & 3.3 & 933.4 \\
\hline
\end{tabular}

\subsection{Edge Illumination and Beam Size Estimate}

Once the position of each horn with respect to the secondary mirror is known, we can use a Gaussian beam approximation to propagate a beam from the horn to the sky to estimate (1) the illumination at the edge of the mirrors and (2) the angular size of the beams in the far field.

For a Gaussian beam, the magnitude of the electric field $E$ in cylindrical coordinates is given by

$$
E(z, \rho)=E_{0} e^{-[\rho / \omega(z)]^{2}},
$$

where $z$ is the axis along the central ray and $\rho$ is the radial distance from the axis. The beam radius $\omega(z)$ is the radius $\rho$ for which $I / I_{0}=1 / e^{2}$. It is given by

$$
\omega(z)=\omega_{0} \sqrt{1+\left(\frac{z}{z_{R}}\right)^{2}},
$$

where

$$
z_{R}=\frac{\pi \omega_{0}^{2}}{\lambda} .
$$

From equation (1), it follows that the intensity $I$ is given by

$$
\frac{I}{I_{0}}=\left(\frac{E}{E_{0}}\right)^{2}=e^{-2[\rho / \omega(z)]^{2}} .
$$

The parameter $\omega_{0}$ is the so-called waist radius, the minimum radius of the $\left(I / I_{0}=1 / e^{2}\right)$ contour of the beam. According to Clarricoats \& Olver (1984), $\omega_{0}=0.64 \times a$, where $a$ is the radius of the aperture of the horn. For the Q-band horns $\omega_{0}=8.6 \mathrm{~mm}$, while for Ka-band horns $\omega_{0}=12.0 \mathrm{~mm}$.

In order to estimate the illumination at the edge of the mirrors, we simulated a Gaussian beam propagating from the horn to the sky. Each time the simulated beam was reflected by a mirror, we calculated the direction and the waist radius of the reflected beam. Table 2 shows the waist radius for each path within the telescope.

Next we defined dummy plane surfaces perpendicular to the central ray and calculated $\omega(z)$ at each of these dummy surfaces and also the distances between the central ray and several points at the edge of the mirrors. Finally, we calculated the illumina-

\begin{tabular}{|c|c|c|}
\hline \multirow[b]{2}{*}{ РАTH } & \multicolumn{2}{|c|}{$\omega_{0}(\mathrm{~mm})$} \\
\hline & $\mathrm{Ka}$ & Q \\
\hline 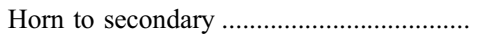 & 12.0 & 8.6 \\
\hline 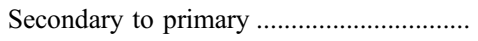 & 10.2 & 7.3 \\
\hline Primary to flat & 486.2 & 488.2 \\
\hline 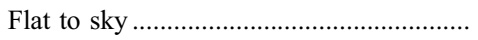 & 486.2 & 488.2 \\
\hline
\end{tabular}
tion using equation (4). We found for the primary mirror that the

TABLE 2

Waist Radius of the Gaussian Beam for Both Bands

\begin{tabular}{|c|c|c|}
\hline $\begin{array}{c}\nu \\
(\mathrm{GHz})\end{array}$ & $\begin{array}{c}\omega \\
(\mathrm{mm})\end{array}$ & $\begin{array}{l}\text { FWHM } \\
\text { (arcmin) }\end{array}$ \\
\hline$\ldots$ & $6.53 \times 10^{3}$ & 26 \\
\hline 41.5 & $4.72 \times 10^{3}$ & 19 \\
\hline
\end{tabular}

TABLE 3

Beam Size (FWHM) at the Far Field

edge illumination level is about $-34 \mathrm{~dB}$ for the central Q-band horn, while for the secondary mirror it is about $-30 \mathrm{~dB}$.

It is worth analyzing equation (4) for two cases: $(i) I / I_{0}=$ $1 / e^{2}$ and $(i i) I / I_{0}=1 / 2$. In the first case $\rho=\omega(z)$, while in the second case $\rho=(\ln 2 / 2)^{1 / 2} \omega(z)$. In the limit when $z \gg z_{R}$,

$$
\tan \theta_{0}=\frac{\omega(z)}{z}
$$

and

$$
\tan (\text { HWHM })=\sqrt{\ln 2 / 2} \tan \theta_{0}=\sqrt{\ln 2 / 2} \frac{\omega(z)}{z} .
$$

Combining these two equations, one finds

$$
\mathrm{FWHM}=2 \mathrm{HWHM}=2 \arctan \left[\sqrt{\ln 2 / 2} \frac{\omega(z)}{z}\right] .
$$

Equation (7) shows that an estimate for the FWHM of the beam can be calculated from both $\omega(z)$ and $z$ on the far field. So we first calculated the beam radius $\omega(z)$ at a plane perpendicular to the central ray and very far from the telescope $\left(z=10^{6} \mathrm{~mm}\right)$ and then we calculated the FWHM. The results are shown in Table 3.

\subsection{Optical Parameters}

The edge illumination and the beam sizes depend on a set of parameters that define the telescope optics. The results previously presented were calculated for the configuration that best satisfies the requirements of the design (Figueiredo 1997). We now describe how these parameters were calculated.

First we defined a set of four parameters that could, in principle, be regarded as arbitrary: (i) focal length of the ellipsoid; (ii) angle between the central ray and the ellipsoid axis; (iii) focal length of the paraboloid; and (iv) maximum size of the primary mirror.

In order to keep the size and mass of the mirrors within the limits of the gondola, the maximum size of the primary was chosen to be $d=2200.0 \mathrm{~mm}$. We have then performed an iterative process to calculate the other three parameters, so that the following requirements would be met:

1. the illumination at the edge of the mirrors should be smaller than $-30 \mathrm{~dB}$;

2. the illumination of any side obstacle near the beam should be smaller than $-30 \mathrm{~dB}$;

3. the beam size should be smaller than $21^{\prime}$ at $41.5 \mathrm{GHz}$ and smaller than $30^{\prime}$ at $30 \mathrm{GHz}$.

Although there is no geometric blockage in the design, we were concerned with a possible undesired effect due to $(i)$ the edge of the secondary near the beam between the primary and the flat mirror and (ii) the edge of the primary near the beam between the flat and the sky. This concern made us take into account requirement 2 above while performing the iterative 
TABLE 4

Parameters that Define the Optics of BeAst

\begin{tabular}{|c|c|}
\hline Parameter & Value \\
\hline \multicolumn{2}{|l|}{ Primary Mirror } \\
\hline Focal length, $q \ldots \ldots$. & $1250.0 \mathrm{~mm}$ \\
\hline Major axis, $d \ldots \ldots \ldots$. & $2200.0 \mathrm{~mm}$ \\
\hline Aperture, $A$ & $1966.1 \mathrm{~mm}$ \\
\hline \multicolumn{2}{|l|}{ Secondary Mirror } \\
\hline Semimajor axis, $a$ & $886.7 \mathrm{~mm}$ \\
\hline Semiminor axis, $b$ & $853.4 \mathrm{~mm}$ \\
\hline 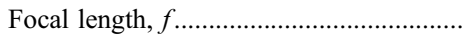 & $240.7 \mathrm{~mm}$ \\
\hline Eccentricity, $e \ldots \ldots \ldots$ & 0.2714 \\
\hline \multicolumn{2}{|l|}{ Flat Mirror } \\
\hline Diameter, $D \ldots \ldots \ldots \ldots . .$. & $2600.0 \mathrm{~mm}$ \\
\hline \multicolumn{2}{|l|}{ Telescope } \\
\hline Feed angle, $2 \alpha$ & $58^{\circ} .2$ \\
\hline Angle between axes, $2 \beta \ldots \ldots \ldots \ldots \ldots \ldots \ldots$ & $35^{\circ} .4$ \\
\hline
\end{tabular}

process. In the final design, the illumination of these potential obstacles were, respectively, -70 and $-100 \mathrm{~dB}$.

After these four parameters were defined, the remaining ones were then calculated from the geometry: (i) semimajor axis of the ellipsoid; (ii) semiminor axis of the ellipsoid; (iii) eccentricity of the ellipsoid; (iv) angle between the axes; and (v) telescope aperture. Table 4 shows the values of the final set of parameters that define BEAST optics.

\section{BEAM PATTERN}

Once we had a design that met our specification, we used GRASP8 software to calculate both the illumination on the mirrors and the beam pattern for each horn. Geometrical optics (GO) and Geometrical theory of diffraction (GTD) have been used on the subreflector, while for the main reflector physical optics (PO) has been applied. Although the use of the GO/GTD does not allow an exact calculation of near field effects, these are expected to be negligible since the typical distance between the feeds and the subreflector is about $900 \mathrm{~mm}$ and the nearfield/far-field transition is at about $400 \mathrm{~mm}$. If $D_{a}$ is the horn diameter ( $\sim 4 \lambda$ for BEAST's horns), the far-field region starts approximately at a distance $d_{\mathrm{FF}} \approx 2 D_{a}^{2} / \lambda \simeq 400 \mathrm{~mm}$. Thus, the use of the GO/GTD leads to the same results as the full PO analysis but in a more efficient calculation time.

Figure 3 shows, for the central horn, both the calculated contour levels on the primary mirror surface and the edge taper, while Figure 4 shows the same for the secondary mirror.

We have also used GRASP8 software to simulate the beam pattern of the telescope. All the calculations have been performed without assuming any focal plane or telescope symmetry. Thus, channels A and B, for example, have been calculated separately. As one would expect, the symmetry in the results agree with the symmetry of the optical design.

In order to calculate the beam patterns on the sky, we have used the usual $(u, v)$ direction cosines given by

$$
u=\sin \theta \cos \phi
$$

and

$$
v=\sin \theta \sin \phi
$$

Each beam has been calculated in a wide $(u, v)$ regular grid between $(-0.087,0.087)$ for both $u$ and $v$, which corresponds to a $10^{\circ} \times 10^{\circ}$ patch on the sky, in order to calculate the position of the beam peak. Once the peak location has been found, a convergency test has been performed and the beams have been calculated in the peak centered coordinate system in a more refined grid of points in the range $(-0.02,0.02)$ both in $u$ and $v$,
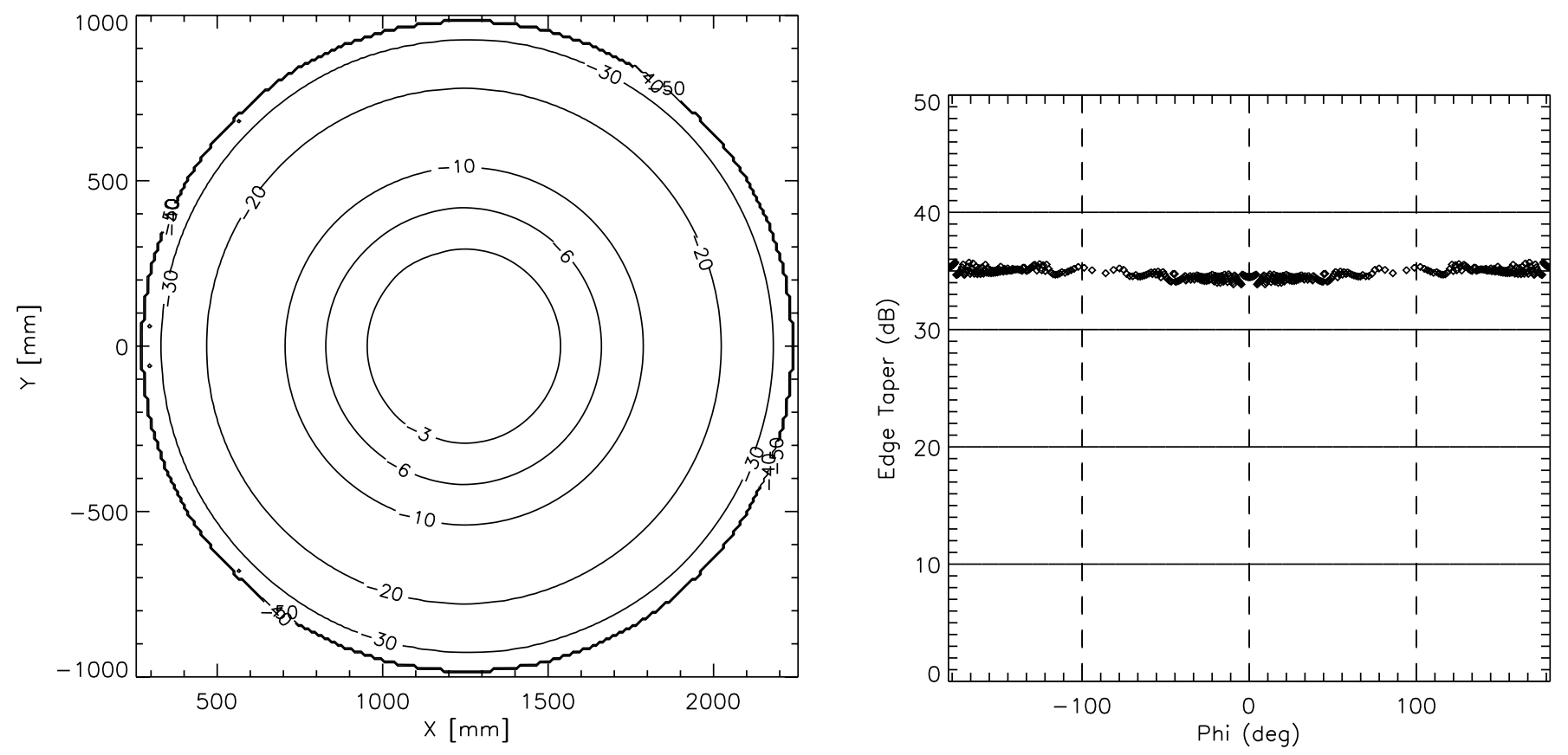

FIG. 3. - Illumination of the primary mirror for the central horn. The left panel shows the contour levels in $\mathrm{dB}$ on the mirror surface, while the right panel shows the edge taper as a function of the azimuthal angle $\phi$. 

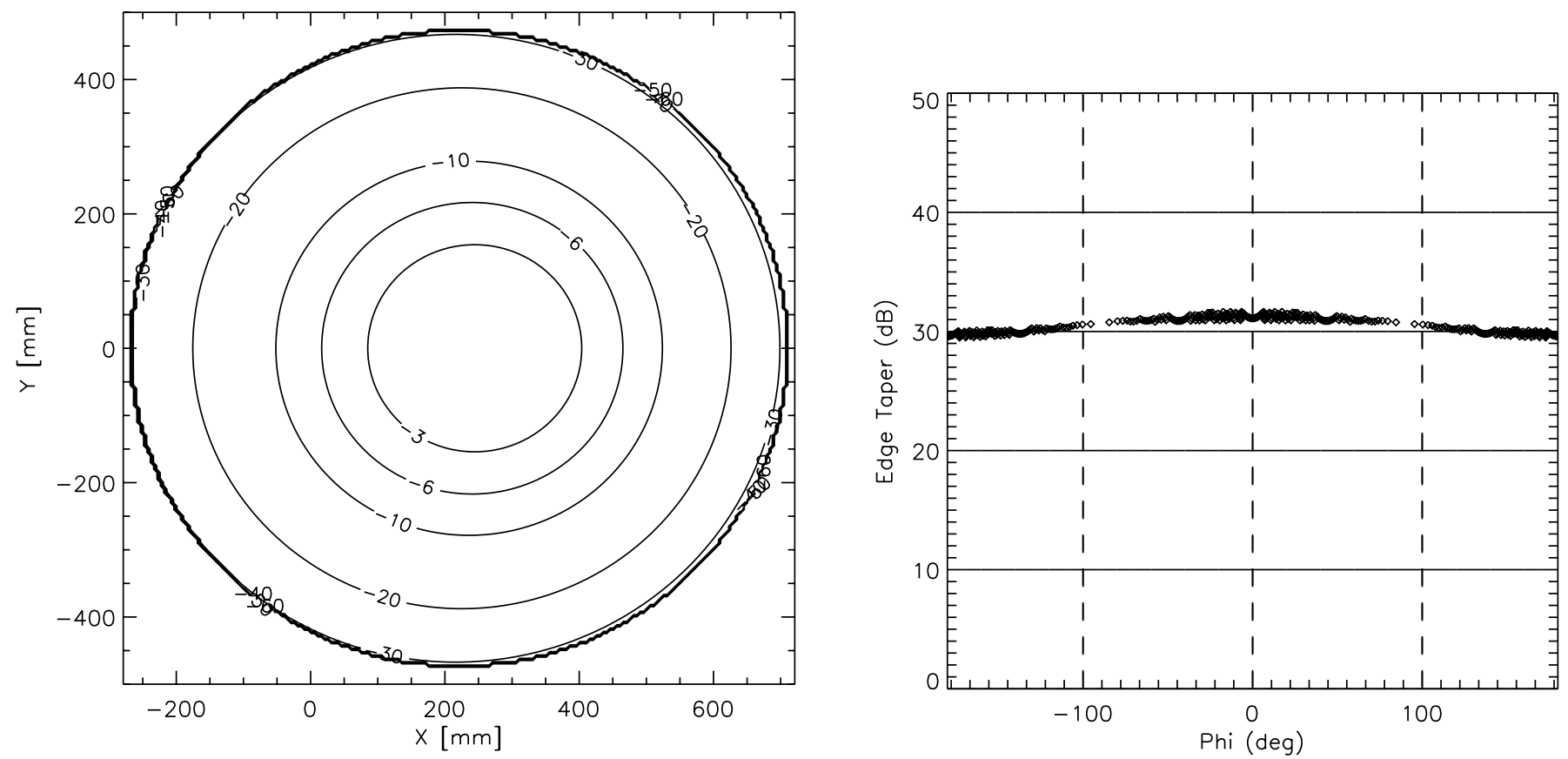

Fig. 4. - Illumination of the secondary mirror for the central horn. The left panel shows the contour levels in $\mathrm{dB}$ on the mirror surface, while the right panel shows the edge taper as a function of the azimuthal angle $\phi$.

which corresponds to a $2^{\circ} .3 \times 2^{\circ} .3$ patch on the sky (Fig. 5). A contour plot of all beams on the $(u, v)$ plane is shown in Figure 6. Table 5 shows the position on the sky of the peak for each beam.

The model of the feed at both frequencies is Gaussian, with an edge taper of -13.89 and $-13.67 \mathrm{~dB}$ at $20^{\circ}$ for the 41.5 and $30.0 \mathrm{GHz}$ channels, respectively.

Because of the aberrations the beam is not circular. Thus, the angular resolution is a function of the azimuthal cut of the beam. We have calculated the maximum and minimum FWHM using

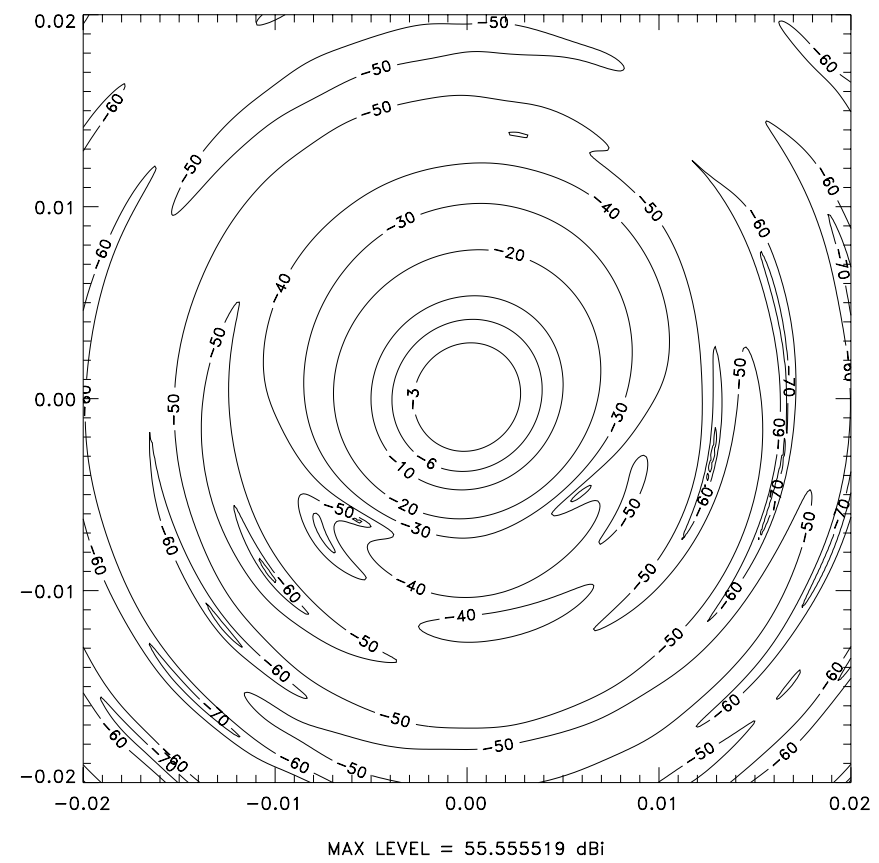

FIG. 5.-Example of the beam calculation (channel A). The calculations have been performed in a frame centered on the peak after the convergency test has been run. The coordinates are the direction cosines $(u, v)$. the GRASP8 post processor. Then we performed an elliptical Gaussian fit of the beam in the $(u, v)$ plane. The average FWHM calculated from the fit is shown in Table 5. We notice that the calculated FWHM agrees with the estimated figures previously shown in Table 3.

The coma lobes seen in Figure 6 do not pose a serious problem for the data analysis because of the observation strategy employed. As we describe in Meinhold et al. (2005), each pixel on the sky is observed several times by all horns, each time under a different orientation.

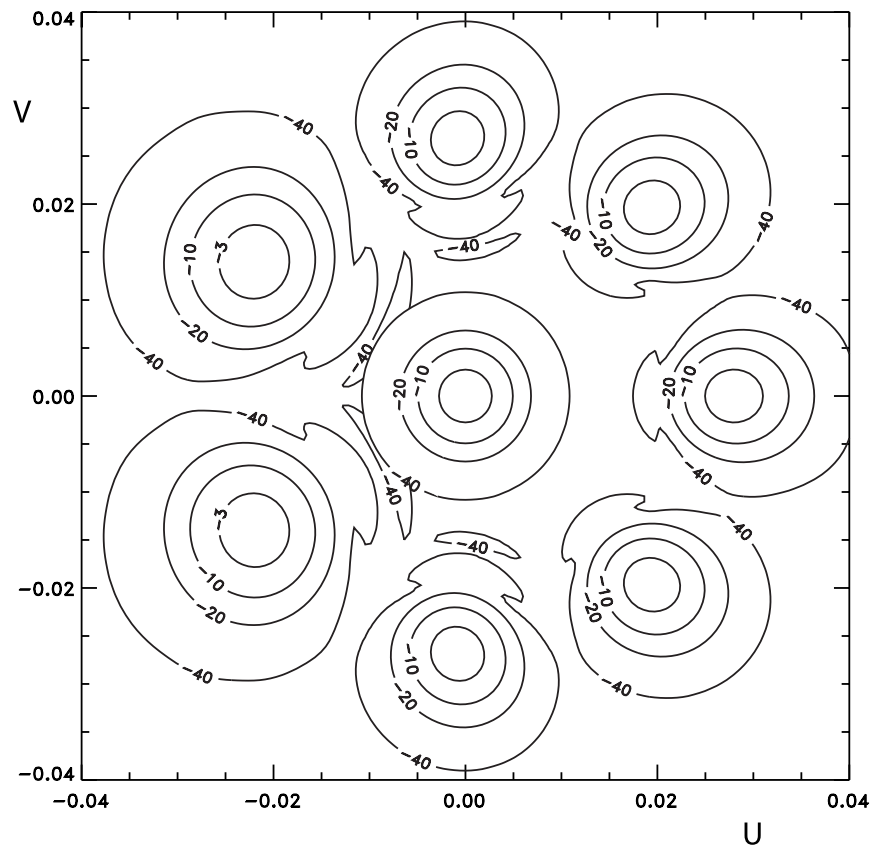

Fig. 6.-Main beam contour plots in a $10^{\circ} \times 10^{\circ}$ patch on the sky. The coordinates are the direction cosines $(u, v)$. 
TABLE 5

Peak Location of the Main Beams in $(u, v)$ Coordinate System

\begin{tabular}{|c|c|c|c|c|}
\hline Horn & $\begin{array}{c}\nu \\
(\mathrm{GHz})\end{array}$ & $\begin{array}{c}u \\
(\mathrm{rad})\end{array}$ & $\begin{array}{c}v \\
(\mathrm{rad})\end{array}$ & $\begin{array}{l}\text { FWHM } \\
(\operatorname{arcmin})\end{array}$ \\
\hline 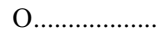 & 41.5 & 0.00000 & 0.00000 & 19.07 \\
\hline A & 41.5 & 0.00082 & 0.02677 & 19.53 \\
\hline $\mathrm{B} \ldots \ldots \ldots \ldots \ldots \ldots$ & 41.5 & -0.00082 & 0.02677 & 19.70 \\
\hline$\ldots \ldots \ldots$ & 41.5 & 0.01936 & 0.01960 & 19.97 \\
\hline $\mathrm{D} \ldots \ldots \ldots \ldots \ldots \ldots \ldots \ldots$ & 41.5 & 0.01936 & -0.01960 & 19.97 \\
\hline E & 41.5 & 0.02789 & 0.00000 & 20.32 \\
\hline $\mathrm{F}$ & 30.0 & -0.02194 & 0.01393 & 25.77 \\
\hline G.... & 30.0 & -0.02194 & -0.01393 & 25.77 \\
\hline
\end{tabular}

The actual beam pattern of each horn was carefully measured using two different targets: the Moon and a Gunn diode source. The results are presented in Childers et al. (2005).

\section{CONCLUSION}

Our results show that we were able to design a relatively compact telescope of the compensated type that meets the very strict requirements of having a narrow beam and an excellent edge illumination for a multiple horn array on the focal plane.
For the current focal plane, the beam sizes are $20^{\prime}$ at $41.5 \mathrm{GHz}$ and $26^{\prime}$ at $30 \mathrm{GHz}$. For the channels to be added soon at 90 and $140 \mathrm{GHz}$, the expected beam sizes are $9^{\prime}$ and $6^{\prime}$, respectively.

We would like to acknowledge the support of CAPES, CNPq (grant 470531/2001-0) and FAPEMIG. T. V. and C. A. W. were partially supported by FAPESP grant 00/06770-2. T. V. was partially supported by CNPq grants 466184/00-0 and 302266/ 88-7-FA. C. A. W. was partially supported by CNPq grant 300409/97-4-FA and FAPESP grant 96/06501-4. J. M. was supported by FAPESP grants $01 / 13235-9$ and $02 / 04871-1$. The development and operations of BEAST were supported by NASA grants NAG5-4078, NAG5-9073, and NAG5-4185, and by National Science Foundation grants 9813920 and 0118297 . In addition we were supported by the White Mountain Research Station, the California Space Institute (CalSpace), and the UCSB Office of Research. Production of the superb BEAST optics were made possible by personal and corporate support from S. Dummer (Surface Optics), J. Wafer and T. Ives (Thin Film Technology), TRW, J. Anthony (Union Carbide), and Able Engineering. Manufacturing of the feeds used in the BEAST experiment were made possible by the work of Enrico Mattaini, Emilio Santambrogio, Pietro Guzzi, and Valerio Muzzini.
Childers, J., et al. 2005, ApJS, 158, 124

Clarricoats, P. J. B., \& Olver, A. D. 1984, Corrugated Horns for Microwave Antennas (London: Peter Peregrinus)

Dragone, C. 1978, Bell System Tech. J., 57, 2663 1981, Bell System Tech. J., 60, 2397

Figueiredo, N. 1997, Ph.D. thesis, Inst. Nacional de Pesquisas Espaciais (INPE), Brazil

\section{REFERENCES}

Meinhold, P. R., et al. 2005, ApJS, 158, 101

Mizuguchi, Y., Akagawa, M., \& Yokoi, H. 1978, Elec. Commun. Japan, 61, 58 Ohtera, I., \& Ujiie, H. 1975, IEEE Trans. Ant. Prop., 23, 858

Scott, D., Silk, J., \& White, M. 1995, Science, 268, 5212 